\title{
Knowledge among drug dispensers and antimalarial drug prescribing practices in public health facilities in Dar es Salaam
}

This article was published in the following Dove Press journal:

Drug, Healthcare and Patient Safety

30 August 2013

Number of times this article has been viewed

\author{
Appolinary AR Kamuhabwa' \\ Richard Silumbe ${ }^{2}$ \\ 'Unit of Pharmacology and \\ Therapeutics, School of Pharmacy, \\ Muhimbili University of Health and \\ Allied Sciences, ${ }^{2}$ Pharmacy Council, \\ Dar es Salaam, Tanzania
}

Background: Irrational prescribing and dispensing of antimalarials has been identified as a contributing factor in the emergence of malaria parasites resistant to existing antimalarial drugs. Factors that contribute to such irrational prescribing and dispensing should therefore be identified to address this problem. The aim of this study was to assess irrational antimalarial drug dispensing and prescribing practices in public health facilities.

Methods: A descriptive-retrospective cross-sectional study was conducted between January and June 2011 in order to assess prescribing and dispensing practices for antimalarial drugs in three public hospitals and nine health centers in Dar es Salaam, Tanzania. Thirty-two drug dispensers were interviewed using a structured questionnaire. A total of 4,320 prescriptions for the period January to December 2010 were collected and assessed for antimalarial drug prescribing patterns.

Results: The majority (84.6\%) of drug dispensers had poor knowledge regarding the basic information required from patients before dispensing artemether-lumefantrine. Seventeen of 32 drug dispensers did not know the basic information that should be given to patients in order to increase absorption of artemether-lumefantrine after oral intake. Most drug dispensers also showed limited knowledge about the dosage and contraindications for artemether-lumefantrine. Eighty-seven percent of all prescriptions contained artemether-lumefantrine as the only antimalarial drug, $77.1 \%$ contained at least one analgesic, and $26.9 \%$ contained at least one antibiotic, indicating unnecessary use of analgesics and antibiotics with antimalarial drugs. A substantial number of prescriptions contained antimalarial drugs that have already been declared ineffective for the treatment of malaria in Tanzania, providing additional evidence of inadequate knowledge among health care workers concerning treatment policy.

Conclusion: Despite the government's efforts to increase public awareness regarding use of artemether-lumefantrine as first-line treatment for uncomplicated malaria, there is still irrational prescribing, dispensing, and use of this combination. Based on the results of this study, it is proposed that regular on-the-job training and continuing education be provided to drug dispensers and prescribers in public health facilities.

Keywords: artemether-lumefantrine, drug dispensers, malaria, prescribing, knowledge

\section{Introduction}

Effective management of malaria requires that the recommended antimalarial medicines are available and used appropriately in the correct formulation, dose, and frequency, and for an adequate duration. Due to failure of effectiveness of antimalarial drugs in most endemic countries, especially for uncomplicated malaria, the World Health Organization recommends that national malaria treatment policies should change from monotherapy to artemisinin-based combination therapies. ${ }^{1}$ In response to this
Kamuhabwa

Unit of Pharmacology and Therapeutics, School of Pharmacy, Muhimbili University of Health and Allied Sciences, PO Box 65013, Dar es Salaam, Tanzania

Tel +255755 576985

Fax +255222150465

Email akamuhabwa@muhas.ac.tz submit your manuscript | www.dovepress.com

Dovepress

http://dx.doi.org/10.2147/DHPS.S50071
Drug, Healthcare and Patient Safety 2013:5 I8I-189

(c) (i) (8) $\odot 2013$ Kamuhabwa and Silumbe. This work is published by Dove Medical Press Ltd, and licensed under Creative Commons Attribution - Non Commercial (unported, v3.0)

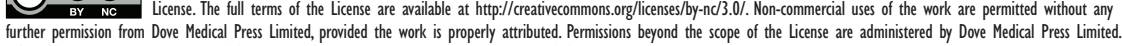
Information on how to request permission may be found at: http://www.dovepress.com/permissions.php 
recommendation, Tanzania changed its malaria treatment policy in 2005, replacing sulfadoxine-pyrimethamine with artemether-lumefantrine as first-line treatment for uncomplicated malaria. ${ }^{2}$ This change is supported by studies from other African countries showing that artemether-lumefantrine is highly efficacious and well tolerated in patients with uncomplicated malaria. ${ }^{1,3}$

A survey conducted in Tanzania in 2007 showed that artemether-lumefantrine was available in more than $75 \%$ of public health facilities. ${ }^{4}$ Availability of artemether-lumefantrine in private and religious health facilities was $50 \%$ and $47 \%$, respectively. This indicates that distribution of artemetherlumefantrine was reaching remote health facilities one year after introduction of the drug into the country. Another study conducted in 2010 showed that artemether-lumefantrine was available in $87 \%$ of private retail pharmacies in Dar es Salaam, ${ }^{5}$ further indicating that the drug is readily available in both public and private health facilities in Tanzania.

Although many countries have developed essential drug lists and treatment guidelines in order to minimize irrational prescribing and dispensing, studies have reported increasingly inappropriate prescribing of antimalarial drugs. ${ }^{6,7}$ These studies have shown that prescribing patterns for medicines are more of the irrational type, including polypharmacy, and overuse of drugs such as antibiotics, injections, and branded rather than generic medicines.

Rational dispensing and prescribing is intended to ensure that the patient gets the medication appropriate for their diagnosis at the correct dose and at the right time. Rational prescribing and dispensing also ensures that there is no unnecessary use of drugs. Polypharmacy reduces patient adherence with medication and may cause unwanted drug-drug interactions, leading to adverse drug reactions. While prescribers are responsible for diagnosis and prescribing of drugs, drug dispensers should ensure that the drugs prescribed are dispensed rationally. Drug dispensers should inform prescribers if there is irrational prescribing, including wrong dosage, potential for drug interactions, and contraindications. Drug dispensers are expected to provide the necessary information to patients about correct use of dispensed drugs at home.

Before introduction of artemether-lumefantrine in Tanzania, a workshop was conducted in October 2006 by the ministry responsible for health in order to train all regional and district drug-dispensing personnel and prescribers working in government-owned hospitals. The purpose of this training was to ensure that drug-dispensing personnel and prescribers were conversant with indications, contraindications, dosage, and use of the drugs. Apart from this training, it is currently not known how effectively this policy has been implemented in terms of rational prescribing and dispensing of artemetherlumefantrine at public health facilities in the country.

A recent study in Dar es Salaam assessing dispensing practices for antimalarial drugs in pediatrics by private retail pharmacies highlighted a lack of knowledge among drug dispensers. ${ }^{5}$ The majority (61\%) of dispensers dispensed antimalarial drugs for pediatric patients without prescription, and about half of them did not consider body weight when determining doses. In general, the findings of this study indicated irrational antimalarial drug dispensing for pediatric patients in private retail pharmacies.

A similar study was conducted in private retail pharmacies to assess dispensing practices of artemether-lumefantrine during pregnancy in Dar es Salaam. ${ }^{8}$ In this study, it was found that only $20 \%$ of drug dispensers knew that artemetherlumefantrine was contraindicated during the first trimester of pregnancy. In addition, about a third of dispensers did not know that artemether-lumefantrine is the drug of choice for treatment of malaria during the second and third trimesters. About a quarter of drug dispensers did not know the correct dosage or duration for artemether-lumefantrine, so gave wrong information and instructions to patients. Given that drug dispensers in private pharmacies were not trained when artemether-lumefantrine was initially introduced, it was assumed that lack of training, continuing education, and proper supervision may have been the reasons for irrational dispensing of antimalarial drugs at these sites.

Lack of knowledge about rational use of antimalarial drugs among prescribers and dispensers is a serious problem, especially in areas of intense transmission, such as Dar es Salaam, where antimalarial drugs are given repeatedly to treat fevers (even in the absence of malaria), thus increasing the risk of resistance and adverse drug reactions. ${ }^{9}$ Due to the number of challenges facing public health facilities in Tanzania, including shortage of qualified health care workers, ${ }^{10}$ it is not known if antimalarial drugs are rationally prescribed or dispensed in these facilities. The shortage of qualified medical personnel and heavy patient loads are more likely to affect rational prescribing and dispensing in public health facilities at the lower levels, including district hospitals, health centers, and dispensaries. In this study, the prescribing and dispensing practices for antimalarial drugs were assessed in district public hospitals and health centers in Dar es Salaam. 


\section{Materials and methods}

\section{Study sites and design}

A descriptive-retrospective cross-sectional study design was used for data collection. A descriptive study design was used to gather information from drug dispensers by interview. Assessment of prescribing practice was done retrospectively by review of outpatient prescriptions available in the health facilities for January to December 2010.

The study was carried out between January and June 2011 in Dar es Salaam, the commercial capital of Tanzania. Administratively, Dar es Salaam is divided into three municipalities, ie, Ilala, Kinondoni, and Temeke. Each municipality has one public hospital and a number of health centers. In total, there are three municipal public hospitals and nine health centers in Dar es Salaam. This study was conducted in all three municipal public hospitals and nine health centers.

\section{Assessment of dispensing practices for antimalarial drugs}

Because of the small number of drug dispensers in the public health facilities, a convenience sampling technique was used to obtain the study sample size. Therefore, this study included all drug dispensers present in the health facilities during the time of the study, ie, seven pharmacists, 16 pharmaceutical technicians, one pharmaceutical assistant, and eight nurses.

In the questionnaire, the scenario of a child with a body weight of $27 \mathrm{~kg}$ was used to assess rational dispensing of artemether-lumefantrine. Items in the questionnaire were designed to assess the knowledge of drug dispensers regarding rational dispensing of artemether-lumefantrine to such a patient, and also to identify the challenges facing drug dispensers in implementation of the national malaria treatment guidelines. ${ }^{2}$

Drug dispensers were asked to indicate the necessary information that should be sought from patients before dispensing artemether-lumefantrine. These included a history of cardiac disease, pregnancy, drug-drug interactions, unwanted effects, and ineffectiveness of artemether-lumefantrine for patients who had used it previously. In addition, drug dispensers were asked to provide the basic information that patients should be given in order to increase absorption of artemetherlumefantrine after oral intake. Knowledge on the part of drug dispensers regarding the dosage of artemether-lumefantrine in terms of number of tablets and frequency of use was also assessed. A knowledge scale was prepared, whereby one point was awarded for each correct answer and a zero point for a wrong answer. The drug dispenser's knowledge was then graded as very poor $(0 \%-24 \%)$, poor $(25 \%-49 \%)$, good $(50 \%-74 \%)$, or excellent $(75 \%-100 \%)$.

\section{Assessment of prescribing practices for antimalarial drugs}

In order to assess prescribing practices for antimalarial drugs in public health facilities, prescriptions for uncomplicated malaria cases were reviewed. In these facilities, prescribers usually prepare antimalarial drug prescriptions for outpatients after assessment of patient signs and symptoms. Depending on the availability of laboratory facilities, the patient may be required to provide peripheral blood for microscopy or a rapid diagnostic test for confirmation of malaria. After prescribing of antimalarial drugs, patients are then directed to collect their medications at the outpatient pharmacy at the health facility. If all medications prescribed are dispensed, the prescription is retained at the pharmacy. In this study, we assessed prescriptions containing antimalarial drugs kept in the outpatient pharmacies in the health facilities for the period of January to December 2010.

The sample size of prescriptions was selected according to World Health Organization guidelines for comparison of drug use involving more than one health facility. ${ }^{11}$ A maximum of 40 prescriptions for uncomplicated malaria cases were collected per month from each health facility for the period January to December 2010. For each health facility, prescriptions were selected using random sampling. During preliminary assessment, it was observed that prescribers worked in shifts at the health facilities. Therefore, in order to collect prescriptions from different prescribers, it was decided to collect the prescriptions at three intervals, ie, in the morning, afternoon, and late evening. A total of 360 prescriptions were collected from each health facility and assessed. Thus, 4,320 prescriptions were collected and assessed for the three municipal public hospitals and nine health centers.

A form was designed to collect information from the prescriptions about antimalarial drug prescribing patterns. It contained information on the total number of drugs prescribed, numbers and names of antimalarial drugs prescribed, the appropriateness of artemether-lumefantrine prescribing, and prescribing of antimalarial drugs together with other drugs, such as analgesics and antibiotics. 


\section{Data management and analysis}

Data in the questionnaire and prescription forms were coded and entered in a computer for analysis using Statistical Package for Social Sciences version 16.0 software (IBM Corporation, Armonk, NY, USA). The total number of drugs per prescription was assessed for each selected health facility. Also, the total number of antimalarial drugs per prescription was calculated. These items were assessed in comparison with the figures recommended in the World Health Organization manual for assessment of drug use. ${ }^{11}$ Using this tool, adherence with national malaria treatment guidelines ${ }^{2}$ was assessed from each health facility. Data analysis was done using descriptive statistics, including frequencies and crosstabulations. The chi-square test was used to calculate $P$-values, which were then used to measure the statistical significance of differences between study variables. A $P$-value $<0.05$ was considered to be statistically significant at the $95 \%$ confidence level.

\section{Ethical considerations}

Drug dispensers were informed about the purpose of the study and asked if they were willing to participate. Written consent was then obtained from study participants. The questionnaire was completed anonymously, and the data were entered into a computer for analysis using code numbers assigned to each questionnaire. Ethical clearance was obtained from the Muhimbili University of Health and Allied Sciences Research and Publications Committee. Permission to conduct the study in the selected health facilities was sought from the municipal medical officers in charge.

\section{Results \\ Dispensing practices for artemether-lumefantrine}

All the pharmacists and eight pharmaceutical technicians interviewed had more than 5 years of working experience. The rest had 1-5 years of working experience. In assessing rational dispensing, questions were formulated to assess the appropriateness of dispensing artemether-lumefantrine to a child with a body weight of $27 \mathrm{~kg}$. Of the 32 dispensers, 22 provided correct information for the recommended doses of artemether-lumefantrine in relation to weight of the patient, while the rest gave wrong information. There was no statistically significance difference $(P=0.115)$ between pharmacists, pharmaceutical technicians, and nurses regarding the importance of patient weight when dispensing artemether-lumefantrine.

The majority (84.6\%) of drug dispensers had poor knowledge regarding the basic information required from patients before dispensing artemether-lumefantrine. The majority were not aware of the contraindications or precautions that should be taken by patients with malaria and cardiovascular disease or pregnant women. Only two pharmacists and four pharmaceutical technicians had good knowledge, while all nurses and pharmaceutical assistants had poor knowledge. Figure 1 shows the knowledge levels of drug dispensers regarding the information that should be sought from patients before dispensing artemether-lumefantrine.

Seventeen (53.1\%) respondents did not know the basic information that patients should be given in order to increase absorption of artemether-lumefantrine after oral intake. Such

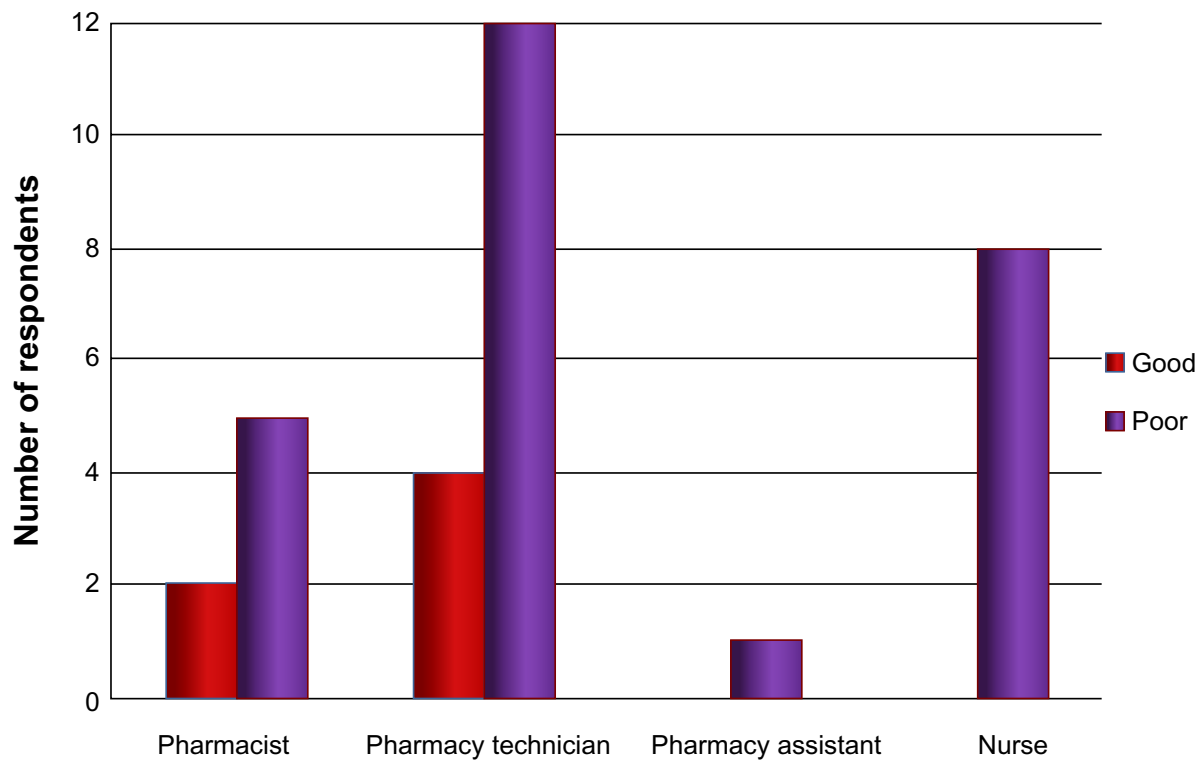

Figure I Knowledge levels of drug dispensers working in public health facilities regarding necessary information that should be sought from patients before dispensing artemether-lumefantrine. 
information includes ingestion of a fatty meal before taking the medicine. In general, pharmaceutical technicians were much more knowledgeable than the rest of the dispensers in this regard. Of the drug dispensers who indicated that milk or a fatty food should be taken before administering artemether-lumefantrine tablets, six (20\%) did not know the importance of taking milk or fatty food. Moreover, most of the respondents were reluctant to recommend milk, fearing that it might affect the efficacy of the medicine if taken concomitantly.

Of the respondents interviewed, 19 reported that they knew the stages of pregnancy during which artemetherlumefantrine could be used. Of these, only seven cited the second and third trimesters as the safe period for use of artemether-lumefantrine during pregnancy. Table 1 shows the results for awareness of drug dispensers regarding use of artemether-lumefantrine during pregnancy. The results show a significant association $(P<0.001)$ between professional training and knowledge about use of artemether-lumefantrine during pregnancy. Pharmacists were more knowledgeable than pharmaceutical technicians and nurses.

Drug dispensers indicated that dispensing of artemetherlumefantrine presents some challenges, including the amount of information that should be given to patients during dispensing. Most patients do not easily understand the information provided, especially concerning dosing intervals. Pack size and number of tablets of artemether-lumefantrine per pack were also mentioned as challenges for dispensing and use of the drug by patients. In particular, use of color coding corresponding to the number of tablets per pack was reported to be confusing for both drug dispensers and patients. Instances were reported of an 18-tablet pack being dispensed to adult patients when the 24-tablet pack was out of stock. Obviously this is an indication of underdosing. The majority of drug dispensers reported having received complaints from patients using artemether-lumefantrine, mainly concerning the complicated dosage regimen and need for long-term use of the medication, thus affecting adherence to the prescribed dosage.

\section{Antimalarial drug prescribing patterns}

Of the 4,320 prescriptions collected from health facilities, it was found that prescribers did not indicate patient gender in $44 \%$ of cases. Details of patient age recorded on the prescriptions showed that $43.4 \%$ were older than 18 years and 45 (1\%) prescriptions did not indicate patient age. About a quarter $(25.6 \%)$ of all prescriptions were for patients aged 6-18 years, while $30 \%$ were for patients younger than 5 years (Table 2).

Figure 2 shows the results for the number of drugs per prescription. The average number of drugs per prescription was $2.4 \pm 0.01$. The maximum number prescribed per prescription was seven, representing about $0.2 \%$ of all prescriptions. The median number of drugs per prescription was two. Of the 4,320 prescriptions assessed, $77.1 \%$ contained at least one analgesic drug, mainly paracetamol or diclofenac. About a quarter (26.9\%) of all prescriptions contained at least one antibiotic.

The majority (98\%) of prescriptions contained only one antimalarial drug. Eighty-seven percent of the prescriptions contained artemether-lumefantrine as the only prescribed antimalarial treatment. A small proportion (3.6\%) of prescriptions contained quinine injection as the only antimalarial drug prescribed, of which $1.9 \%$ were for quinine tablets and $0.7 \%$ contained both quinine injection and tablets. About $5 \%$ of prescriptions contained other antimalarial medicines, including artemether injection, amodiaquine, dihydroartemisininpiperaquine (Duo-cotecxin ${ }^{\circledR}$; Holley Pharmaceuticals, Tustin, CA, USA), sulfamethoxpyrazine-pyrimethamine (Metakelfin ${ }^{\circledR}$; Pfizer, New York, NY, USA), and sulfadoxinepyrimethamine (Fansidar ${ }^{\circledR}$; Roche, Basel, Switzerland) (Table 3). Only $3.1 \%$ of prescriptions contained the correct prescribing information for artemether-lumefantrine. The assessment was based on written information, including timing between doses and duration of drug use.

\section{Discussion}

Because of widespread resistance of Plasmodium falciparum to commonly used antimalarial monotherapies, particularly

Table I Awareness of drug dispensers working in public health facilities regarding use of artemether-lumefantrine during pregnancy

\begin{tabular}{|c|c|c|c|c|c|c|}
\hline \multirow{2}{*}{$\begin{array}{l}\text { Professional category } \\
\text { of respondent }\end{array}$} & \multicolumn{6}{|c|}{ Safe period for use of artemether-lumefantrine during pregnancy } \\
\hline & $\begin{array}{l}\text { First } \\
\text { trimester }\end{array}$ & $\begin{array}{l}\text { Second } \\
\text { trimester }\end{array}$ & $\begin{array}{l}\text { Third } \\
\text { trimester }\end{array}$ & $\begin{array}{l}\text { Both second and } \\
\text { third trimesters }\end{array}$ & Do not know & Total \\
\hline Pharmacist & 0 & 0 & 0 & 6 & 1 & 7 \\
\hline Pharmaceutical technician & 2 & I & 3 & 5 & 5 & 16 \\
\hline Pharmaceutical assistant & 0 & I & 0 & 0 & 0 & I \\
\hline Nurse & 0 & 0 & 1 & 0 & 7 & 8 \\
\hline Total & $2(6.3 \%)$ & $2(6.3 \%)$ & $4(12.5 \%)$ & II (34.4\%) & I3 (40.6\%) & $32(100 \%)$ \\
\hline
\end{tabular}


Table 2 Sociodemographic characteristics of patients recorded in antimalarial drug prescriptions in public health facilities $(\mathrm{n}=4320)$

\begin{tabular}{lll}
\hline Sociodemographic characteristics & $\mathbf{n}$ & Percentage \\
\hline Sex & & \\
Male & $\mathrm{I}, 189$ & 27.5 \\
Female & $\mathrm{I}, 23 \mathrm{I}$ & 28.5 \\
$\quad$ Not indicated & $\mathrm{I}, 900$ & 44 \\
Age group (years) & & \\
0-5 & $\mathrm{I}, 294$ & 30 \\
6-18 & $\mathrm{I}, 104$ & 25.6 \\
Over I8 & $\mathrm{I}, 877$ & 43.4 \\
Not indicated & 45 & $\mathrm{I}$ \\
Total & 4,320 & 100 \\
\hline
\end{tabular}

chloroquine and sulfadoxine-pyrimethamine, Tanzania adopted artemisinin-based combination therapy as the firstline regimen for treatment of uncomplicated malaria in $2006 .{ }^{2}$ In the same year, in an effort to ensure that artemetherlumefantrine is prescribed, dispensed, and used rationally, the government provided training to prescribers and drug dispensers in public health facilities via the Ministry of Health and Social Welfare.

Because of its pharmacokinetic profile, artemetherlumefantrine needs a loading dose, such that the second dose is taken 8 hours after the first dose, and the four subsequent doses are taken 12 hourly. Given that training was provided in public health facilities about the use of artemether- lumefantrine in 2006, it was expected that drug dispensers in these premises would be aware of the correct dosage for this agent. However, the findings of this study show that more than two-thirds of drug dispensers in public health facilities had poor knowledge regarding the recommended dose and dosage schedules for artemether-lumefantrine. Consequently, drug dispensers gave wrong information and instructions to patients about the use of this treatment. Similar findings were recently reported among drug dispensers working in private retail pharmacies in Dar es Salaam. ${ }^{8}$ This is an indication that further training should be provided to drug dispensers regarding the rational dispensing of antimalarial drugs.

It is known that artemether-lumefantrine should be used carefully by pregnant women and in patients with cardiovascular disease. ${ }^{12,13}$ In this study, $84.6 \%$ of the drug dispensers interviewed were not aware of the contraindications for artemether-lumefantrine. As a result, drug dispensers were unable to counsel specific groups of patients about use of the drug, including those with cardiovascular problems, pregnant women, and children. Unless the benefits outweigh the risks, artemetherlumefantrine is contraindicated during the first trimester of pregnancy. ${ }^{12,13}$ These findings necessitate the need to provide continuing education among drug dispensers regarding use of antimalarials in pregnancy and in other groups of patients. ${ }^{5,8}$

In this study, $20 \%$ of drug dispensers did not know the importance of fatty meals when administering artemetherlumefantrine, so it is unlikely that drug dispensers would

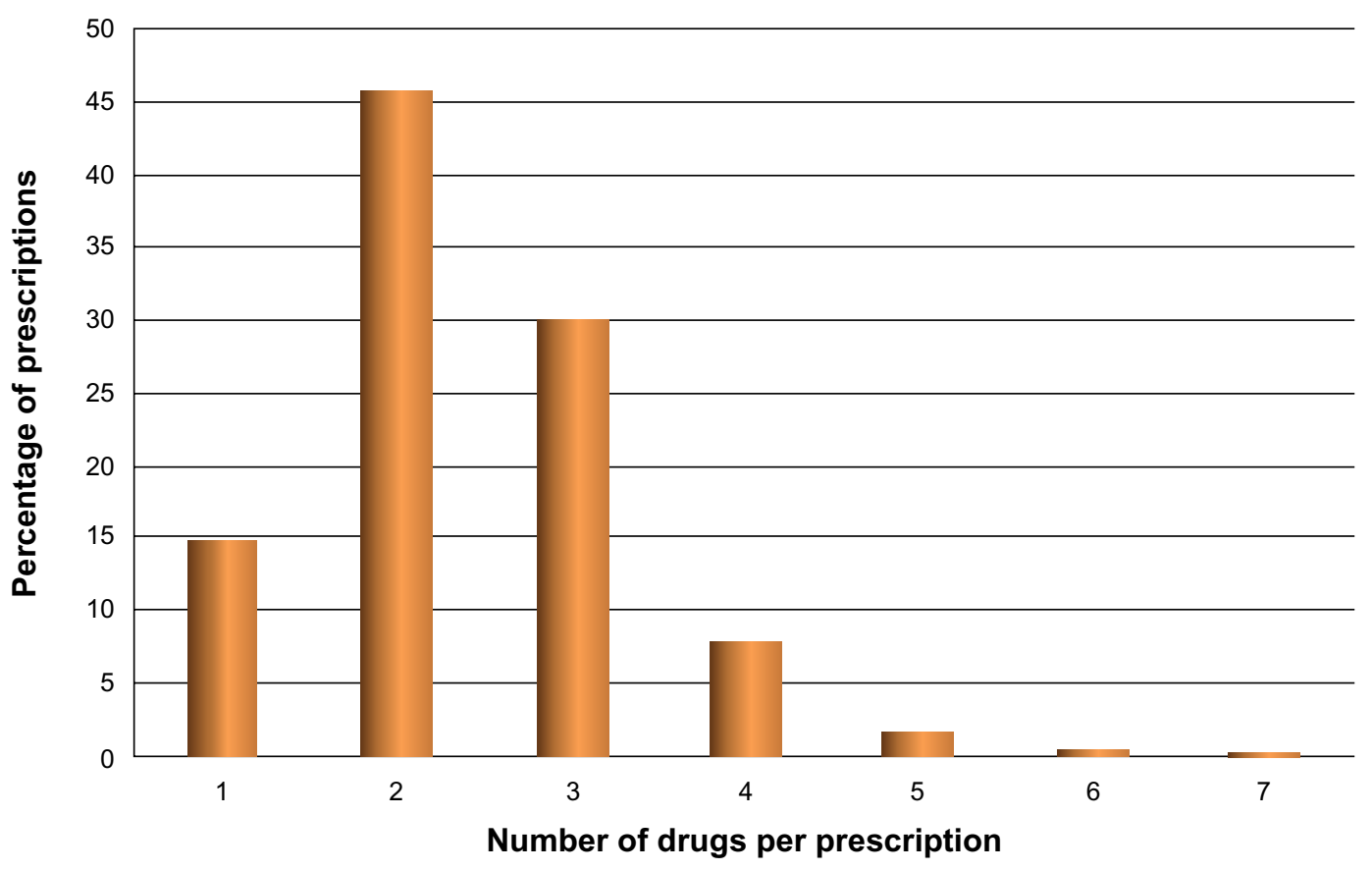

Figure 2 Number of drugs per prescription obtained from public health facilities between January and December 2010 ( $n=4,320$ prescriptions). 
Table 3 Numbers and names of antimalarial drugs per prescription obtained from public health facilities between January and December 2010 ( $n=4,320$ prescriptions)

\begin{tabular}{|c|c|c|c|c|c|c|}
\hline \multirow{2}{*}{$\begin{array}{l}\text { Antimalarial drugs } \\
\text { per prescription (n) }\end{array}$} & \multicolumn{6}{|c|}{ Antimalarial drug prescribed } \\
\hline & $\begin{array}{l}\text { Artemether- } \\
\text { lumefantrine }\end{array}$ & $\mathbf{S P}^{\mathbf{a}}$ & Quinine tablets & Quinine injection & $\begin{array}{l}\text { Quinine injection } \\
\text { and tablets }\end{array}$ & Others $^{b}$ \\
\hline I & 3,765 (99.9\%) & 59 (92.2\%) & $83(100 \%)$ & $156(100 \%)$ & $26(81.3 \%)$ & I 46 (67.9\%) \\
\hline 2 & $5(0.1 \%)$ & $5(7.8 \%)$ & $0(0 \%)$ & $0(0 \%)$ & $6(18.8 \%)$ & $69(32.1 \%)$ \\
\hline Total & $3,770(87.3 \%)$ & $64(1.5 \%)$ & $83(1.9 \%)$ & 156 (3.6\%) & $32(0.7 \%)$ & $215(5 \%)$ \\
\hline
\end{tabular}

Notes: aSulfamethoxpyrazine-pyrimethamine or sulfadoxine-pyrimethamine; 'others (including dihydroartemisinin-piperaquine, artemether injection, and amodiaquine).

advise patients correctly about the benefits of taking the drug with meals. After oral administration, peak plasma artemether concentrations typically occur within 2 hours and peak lumefantrine concentrations are reached in 6-8 hours. Administration with fat-containing foods, including milk, improves bioavailability by more than two-fold for artemether and up to 16-fold for lumefantrine in adults. ${ }^{2}$ For this reason, taking artemether-lumefantrine on an empty stomach is likely to reduce the absorption of lumefantrine substantially, leading to suboptimal plasma drug concentrations, with potential for resistance of $P$. falciparum to the drug. Therefore, to derive maximum effect of artemether-lumefantrine, it is necessary to advise patients to take the drug with fatty meals.

A number of challenges were mentioned by drug dispensers with regard to dispensing and use of artemetherlumefantrine. Notable challenges included too much patient information being required and patients being unable to understand the instructions for use of the drug at home. Drug dispensers are responsible for advising patients on all matters pertaining to adherence with a treatment schedule and proper drug use. However, due to inadequate knowledge on the part of drug dispensers concerning correct use of artemether-lumefantrine, patients are not correctly advised. Poor knowledge among drug dispensers regarding dosage, contraindications, drug-food interactions, and side effects may contribute to the negative perception of many patients toward use of this drug. ${ }^{8}$

A study of dispensaries and private pharmacies in Dar es Salaam in 2011 indicated that health care workers in dispensaries were much more knowledgeable than drug dispensers in private pharmacies. ${ }^{14} \mathrm{~A}$ similar study in Dar es Salaam also confirmed low levels of knowledge among drug dispensers in private community pharmacies. ${ }^{8}$ The observed differences between the findings of the present study and that of previous research ${ }^{14}$ regarding knowledge among health care workers in public health facilities are mainly due to the different levels of health facilities assessed in these studies. In the earlier study, ${ }^{14}$ assessments were done in dispensaries, which are the lowest health care facility level in Tanzania. Moreover, in our study, the knowledge of health care workers was assessed differently. Whereas drug dispensers were interviewed, knowledge among prescribers was assessed by review of antimalarial drug prescriptions over a period of one year. In addition, the knowledge levels were set at different categories after considering all the necessary parameters for correct prescribing and dispensing of artemether-lumefantrine. From these findings, it can be concluded that health care workers at different levels of the health care system in Tanzania have inadequate knowledge about the use of artemether-lumefantrine for treatment of uncomplicated malaria.

In assessing antimalarial prescribing patterns in public health facilities, several parameters were evaluated. These included the average number of medicines per prescription, average number of analgesics, and average number of antibiotics prescribed with antimalarial drugs. In this study, the average number of medicines (2.4) per prescription was found to be slightly lower than that reported by other studies conducted in Yemen, ${ }^{15}$ Nigeria,,${ }^{16}$ and the United Arab Emirates. ${ }^{17}$ However, the average number of medicines per prescription in this study is slightly higher than that reported in Dubai. ${ }^{18}$

In a study from Uganda, it was reported that the likelihood of being prescribed antibiotics increased in patients with febrile illness who tested negative for malaria. ${ }^{19}$ Other studies have reported similar findings, indicating compensatory antibiotic prescription in febrile patients with negative results for malaria. ${ }^{20,21}$ These findings are in agreement with the results of the present study, in which antibiotics were found to be prescribed without an indication of bacterial infection as the cause of fever. The shortage of qualified health care workers, ${ }^{10}$ inadequate laboratory facilities, ${ }^{22}$ and lack of continuing education for health care workers are among the reasons for irrational prescribing in public health facilities.

An interesting observation is that a substantial number of prescriptions contained antimalarial drugs that have already 
been declared ineffective for the treatment of malaria in Tanzania. This observation provides additional evidence of inadequate knowledge of prescribers regarding treatment policy. ${ }^{23}$ In addition, only $3.1 \%$ of prescriptions contained correct information about artemether-lumefantrine, indicating that both prescribers and drug dispensers have inadequate knowledge about the policy for treatment of malaria in Tanzania. Moreover, because supply of medicines from the medical stores department is often inadequate, most health facilities procure some medicines, including antimalarial drugs, from private suppliers. This is a main source of antimalarial drugs known to be ineffective for the treatment of malaria. Therefore, there is a need for the food and drugs authority in Tanzania to ensure that drugs, which are no longer effective for the treatment of malaria, are not registered and removed from the market.

In terms of patient information recorded in the prescription, the results of this study show that the percentage of prescriptions without an indication of patient age was much lower than that reported in Yemen, ${ }^{15}$ the United Arab Emirates, ${ }^{17}$ and Dubai. ${ }^{18}$ On the other hand, the percentage of prescriptions (44\%) without information on patient gender was much higher than that reported in public hospitals and health centers in Yemen. ${ }^{15}$ These results indicate a need to promote formal prescription writing among prescribers in health facilities, so as to enhance rational antimalarial dispensing and drug use.

To optimize the benefits of drug expenditure by governments and patients, it is necessary to promote rational drug use, including selection of drugs recommended on the essential drug list. The findings of our study show that artemether-lumefantrine was prescribed first-line for uncomplicated malaria in most (89\%) prescriptions for antimalarial drugs. A similar study from Nigeria showed that $58.1 \%$ of prescribers did not adhere to the national malaria treatment policy. ${ }^{16}$ However, our study shows that there is still a pattern of prescribing other antimalarial drugs, such as sulfamethoxpyrazine-pyrimethamine, artemether injection, amodiaquine, sulfadoxine-pyrimethamine, and dihydroartemisinin-piperaquine. With increasing resistance of malarial parasites to most existing antimalarial drugs, these findings highlight a need for aggressive promotion of prescribing the recommended artemisinin combination therapies for management of uncomplicated malaria.

\section{Conclusion}

This study has identified irrational dispensing and prescribing of artemether-lumefantrine in public health facilities. Drug dispensers do not have sufficient knowledge regarding dosage, drug-drug interactions, drug-food interactions, and contraindications for artemether-lumefantrine. An excessive number of drugs are prescribed per patient, with unnecessary prescribing of analgesics and antibiotics. These findings highlight the challenges that exist in ensuring rational use of antimalarial drugs. Regular on-the-job training and short courses for drug dispensers and prescribers are recommended for more rational use of antimalarial drugs. The food and drugs authority in Tanzania should enforce regulations ensuring availability of the recommended antimalarial drugs, and phase out drugs to which malaria parasites have shown high resistance.

\section{Acknowledgments}

We acknowledge the financial support provided by Muhimbili University of Health and Allied Sciences. We would also like to express our sincere gratitude to the medical officers in charge of the municipalities for granting permission to carry out the study in their hospitals and health centers. We thank all the drug dispensers for participating in the interviews and providing access to their antimalarial drug prescriptions. We also wish to thank the research assistants for their time and effort during data collection.

\section{Disclosure}

The authors report no conflicts of interest in this work.

\section{References}

1. Ministry of Health and Social Welfare. National guidelines for malaria diagnosis and treatment. Malaria Control Series 11. Dar es Salaam, Tanzania: Ministry of Health and Social Welfare; 2006. Available from: http://apps.who.int/medicinedocs/documents/s19271en/s19271en.pdf. Accessed July 20, 2013.

2. World Health Organization Regional Office for Africa. A strategic framework for malaria prevention and control during pregnancy in the African region, 2004. Available from: http://www.who.int/malaria/ publications/atoz/afr_mal_04_01/en/. Accessed July 20, 2013.

3. Steketee RW, Nahlen BL, Parise ME, Menendez C. The burden of malaria in pregnancy in malaria-endemic areas. Am J Trop Med Hyg. 2001;64:28-35.

4. Ministry of Health and Social Welfare. Tanzania drug tracking study. Final report, 2007. Available from: http://apps.who.int/medicinedocs/ documents/s19982en/s19982en.pdf. Accessed July 20, 2013.

5. Kamuhabwa A, Ramji K. Antimalarial drugs for pediatrics: prescribing and dispensing practices in Tanzanian City. Trop J Pharm Res. 2011;10: 611-618.

6. Yousif MA, Adeel AA. Antimalarials prescribing patterns in Gezira State: precepts and practices. East Mediterr Health J. 2000;6:939-947.

7. Bhartiy SS, Shinde M, Nandeshwar S, Tiwari SC. Pattern of prescribing practices in the Madhya Pradesh, India. Kathmandu Univ Med J. 2008;6: 55-59.

8. Kamuhabwa A, Mnyusiwalla F. Knowledge of drug dispensers and pregnant women on the use of artemether-lumefantrine during pregnancy. Tanzania J Health Res. 2011;13:108-115.

9. Whitty CJ, Rowland M, Sanderson F, Mutabingwa TK. Malaria. BMJ. 2002;325:1221-1224. 
10. Ministry of Health and Social Welfare. United Republic of Tanzania. Human resources for health strategic plan 2008-2013. Available from: http://www.unfpa.org/sowmy/resources/docs/library/R223_MOHTanzania_2008_HRH_Strategic_Plan_2008_2013.pdf. Accessed July 20, 2013.

11. World Health Organization. How to investigate drug use in health facilities: selected drug use indicators. Geneva: World Health Organization; 1993. Available from: http://apps.who.int/medicinedocs/pdf/s2289e/ s2289e.pdf. Accessed August 8, 2013.

12. Clark R, Kumemura M, Makori N, et al. Artesunate: developmental toxicity in monkeys. Abstract presented at the 46th annual meeting of the Teratology Society, Tucson, AZ, June 24-29, 2006.

13. Dellicour S, Hall S, Chandramohan D, Greenwood B. The safety of artemisinins during pregnancy: a pressing question. Malar $J$. 2007;6:15.

14. Mugoyela V, Minzi O. Implementation of artemether-lumefantrine policy for malaria at health facilities in Tanzania. Risk Manag Healthc Policy. 2011;4:89-95.

15. Abbo-Raddo A. Prescribing rationality and availability of antimalarial drugs in Hajjah, Yemen. East Mediterr Health J. 2003;9:4.

16. Adebayo ET, Hussain NA. Pattern of prescription drug use in Nigerian army hospitals. Ann Afr Med. 2010;9:152-158.

17. Bazigha K, Sahar A, Eman F, Heyam S. Professional practices and perception towards rational use of medicines according to WHO methodology in United Arab Emirates. J Pharm Pract. 2010;8:70-76.
18. Sharif SI, Al-Shaqra M, Hajjar H, Shamout A, Wess L. Pattern of drug prescribing in a hospital in Dubai, United Arab Emirates. Libyan J Med. 2008;3:10-12.

19. Batwala V, Magnussen $P$, Nuwaha F. Antibiotic use among patients with febrile illness in a low malaria endemicity setting in Uganda. Malar J. 2011;10:377.

20. Reyburn H, Mbakilwa H, Mwangi R, et al. Rapid diagnostic tests compared with malaria microscopy for guiding outpatient treatment of febrile illness in Tanzania: randomized trial. BMJ. 2007;334:403.

21. D'Acremont V, Kahama-Maro J, Swai N, Mtasiwa D, Genton B, Lengeler C. Reduction of anti-malarial consumption after rapid diagnostic tests implementation in Dar es Salaam: a before-after and cluster randomized controlled study. Malar J. 2011;10:107.

22. Kamuhabwa A, Chavda R. Health care provider's preparedness for H1N1/09 flu prevention and treatment in Dar es Salaam, Tanzania. J Infect Dev Ctries. 2012;6:262-270.

23. Watsierah CA, Onyango RO, Ombaka JH, Abong'o BO, Ouma C. Provider knowledge of treatment policy and dosing regimen with artemether-lumefantrine and quinine in malaria-endemic areas of eastern Kenya. Malar J. 2012;11:436
Drug, Healthcare and Patient Safety

\section{Publish your work in this journal}

Drug, Healthcare and Patient Safety is an international, peer-reviewed open-access journal exploring patient safety issues in the healthcare continuum from diagnostic and screening interventions through to treatment, drug therapy and surgery. The journal is characterized by the rapid reporting of reviews, original research, clinical, epidemiological and

\section{Dovepress}

post-marketing surveillance studies, risk management, health literacy and educational programs across all areas of healthcare delivery. The manuscript management system is completely online and includes a very quick and fair peer-review system. Visit http://www.dovepress.com/ testimonials.php to read real quotes from published authors. 